\title{
Sensor Placement via Optimal Experiment Design in EMI Sensing of Metallic Objects
}

\author{
Lin-Ping Song, ${ }^{1}$ Leonard R. Pasion, ${ }^{2}$ Nicolas Lhomme, ${ }^{2}$ and Douglas W. Oldenburg ${ }^{1}$ \\ ${ }^{1}$ Department of Earth, Ocean and Atmospheric Sciences, University of British Columbia, Vancouver, Canada \\ ${ }^{2}$ Black Tusk Geophysics Inc., Vancouver, Canada \\ Correspondence should be addressed to Lin-Ping Song; lpsong@eos.ubc.ca
}

Received 12 July 2016; Revised 19 October 2016; Accepted 1 November 2016

Academic Editor: Sebastian Heidenreich

Copyright (C) 2016 Lin-Ping Song et al. This is an open access article distributed under the Creative Commons Attribution License, which permits unrestricted use, distribution, and reproduction in any medium, provided the original work is properly cited.

\begin{abstract}
This work, under the optimal experimental design framework, investigates the sensor placement problem that aims to guide electromagnetic induction (EMI) sensing of multiple objects. We use the linearized model covariance matrix as a measure of estimation error to present a sequential experimental design (SED) technique. The technique recursively minimizes data misfit to update model parameters and maximizes an information gain function for a future survey relative to previous surveys. The fundamental process of the SED seeks to increase weighted sensitivities to targets when placing sensors. The synthetic and field experiments demonstrate that SED can be used to guide the sensing process for an effective interrogation. It also can serve as a theoretic basis to improve empirical survey operation. We further study the sensitivity of the SED to the number of objects within the sensing range. The tests suggest that an appropriately overrepresented model about expected anomalies might be a feasible choice.
\end{abstract}

\section{Introduction}

Unexploded ordnance (UXO) refers to malfunctioned, undetonated military munitions that are present at former war sites and military practice ranges. UXOs pose a serious problem worldwide on public and environmental safety due to their high-risk explosion threats to human life and potential leaching of toxic explosive chemicals into soil and groundwater system $[1,2]$. In light of these concerns, the cleanup of UXO contaminated sites has drawn significant attention over the past two decades. The fundamental cleanup work requires to detect buried UXO at depth and to separate UXO from the vast amount of innocuous metallic debris. Geophysical sensing techniques based on electromagnetic induction (EMI) have been found to be the most effective for detecting and characterizing shallow UXOs [3].

An EMI sensing system consists of transmitter and receiver coils. The transmitter coil emits a primary magnetic field to illuminate the subsurface. A time varying primary field induces eddy currents in a nearby metallic object. By Faraday's law [4], these induced currents produce a transient secondary magnetic field that can be measured by a receiver. Provided that the target dimension is small relative to the target-sensor distance and the primary fields around the target are approximately uniform, the transient scattering phenomena of a metal target can generally be well described by an equivalent induced dipole $[4,5]$. Under this scattering model, the induced principal dipole polarizabilities are designated as the target signature for classification since they are intrinsic function of the geometry and material properties of an object. The principal polarizability characteristic of UXOs exhibits one strong axial polarizability and two equal weaker transverse polarizabilities [6-10]. That is the fundamental physical attribute used for differentiating potential UXOs from metallic debris in the EMI sensing classification approach. To reliably classify UXO requires accurate estimates of dipole polarizabilities from the geophysical inversion of EMI data [6-10].

EMI data are collected in either a dynamic mode or cued mode [3]. In a dynamic mode, the sensor is moved across the area of interest to map data anomalies that indicate metallic contamination. In a cued survey mode, the sensor is placed stationarily at locations of data anomalies identified in the dynamic survey. The cued survey helps to acquire high 
signal-to-noise ratio (SNR) data by increased stacking. In addition, instrument position errors are minimized. Therefore, a followup cued survey is often used to acquire data for improving polarizability estimates.

In this paper, we focus on the sensor placement problem in cued survey. For sites where anomalies are well separated, the current practice of cued sounding likely positions a sensor directly over a nearby target. For some circumstances the instrument might be repositioned above the target by inspecting the offset between the sensor location and the estimated location of an assumed single target. In this way, the SNR of cued data is maximized as possible. For sites with high anomaly density, we likely encounter instances where more targets are present in the field of view of the instrument. The objects near the sensor-edge might be poorly characterized due to the signal being dominated by objects closest to the center of the sensor. Additional soundings may be desired to better characterize the anomalies. Ideally, the addition of new data would reduce model uncertainty. The extent to which model uncertainty is reduced depends on the sensing locations. Therefore, we can treat adding soundings as an optimal experimental design (OED) problem $[11,12]$ in which optimal sensor positions are predicted in order to collect the most informative data for accurately estimating model parameters.

The theory of optimal experimental design has been well documented in statistics and extensively applied to various scientific and engineering fields [11, 12]. The OED theory contains design and model parameters. In the context of EMI sensing of UXO, the design parameter refers to the number and locations of sensors that control the survey quality. The model parameters refer to locations and polarizabilities of targets (see a brief review of the signal model in Section 2). An optimal survey design is implemented using statistical criterion $[11,12]$. A common choice is the $D$-criterion that minimizes the determinant of the model covariance matrix. The determinant in the $D$-criterion is a function of both design and model parameters. When the true model parameters are known or specified, it can be relatively straightforward to find design parameters. For the EMI classification problem, we face a nonlinear optimization problem where optimizing design parameters depends on the model parameters that are unknown before the survey. To make the problem tractable, we consider a sequential design procedure $[13,14]$ that forecasts a new sensor placement using estimated model parameters given an initial/current survey. We then update model parameters using additional new data. This procedure is repeated until a stopping criterion is satisfied. A stopping criterion could be a predefined threshold of information gain or a maximum number of sensing.

The remaining parts of this paper are organized as follows. In Section 2, we describe the data model which briefly reviews the physics of the EMI sensing. In Section 3, the linearized model and the associated optimality design criteria are discussed. Then the sensor placement problem is formulated as a sequential experimental design (SED) process. In Section 4, the results from the synthetic and experimental data are presented to evaluate the SED technique. Section 5 summarizes the paper.

\section{Data Model}

Consider a time domain electromagnetic system that is deployed near the surface to interrogate a buried metallic object. When the dimension of a target is small relative to the target-sensor distance, the low frequency EMI scattering of a metal target can be well represented by an equivalent induced dipole model $[6,7,15,16]$. Mathematically, a target is characterized by the $3 \times 3$ magnetic polarizability tensor $P(t)$ at time $t$ :

$$
P(t)=\left[\begin{array}{lll}
p_{11}(t) & p_{12}(t) & p_{13}(t) \\
p_{21}(t) & p_{22}(t) & p_{23}(t) \\
p_{31}(t) & p_{32}(t) & p_{33}(t)
\end{array}\right]
$$

The elements of the tensor $p_{i j}(t)$ represent a dipole component in the $i$ th Cartesian direction due to a primary field in the $j$ th Cartesian direction. The polarizability tensor is symmetric and positive definite. The principal polarizabilities $L_{i}(t)$ are obtained through an eigen decomposition

$$
P(t)=\sum_{i=1}^{3} L_{i}(t) \mathbf{e}_{i} \mathbf{e}_{i}^{T},
$$

where $\mathbf{e}_{i}$ is the orthonormal eigenvector representing the $i$ th principal direction of dipolar polarization with respect to a reference system. The principal polarization $L_{i}(t)$ are a function of the target's size, shape, and material properties (e.g., magnetic susceptibility and electric conductivity).

Assuming a time domain EMI system consisting of $M$ transmitter-receiver coil pairs, we can express the corresponding measurements as [10]

$$
\mathbf{d}(t)=\sum_{k=1}^{\eta} A\left(\mathbf{r}_{k}, \mathbf{r}_{s}\right) \mathbf{q}_{k}(t)+\mathbf{n}(t),
$$

where $\mathbf{d}(t)=\left[d_{1}(t), \ldots, d_{M}(t)\right]^{T}$ is an $M \times 1$ measured data vector at time $t, \eta$ is the number of targets in the field of view of the sensors, $A\left(\mathbf{r}_{k}, \mathbf{r}_{s}\right)$ is an $M \times 6$ matrix denoting the sensitivities of the sensor system at $\mathbf{r}_{s}$ to the $k$ th object located at $\mathbf{r}_{k}, \mathbf{q}_{k}(t)$ is a $6 \times 1$ column vector whose components are the elements of the polarizability tensor $P_{k}(t)$ of the $k$ th object, and $\mathbf{n}(t)$ is the additive noise vector. They are given by

$$
\begin{aligned}
A\left(\mathbf{r}_{k}, \mathbf{r}_{s}\right) & =\left[\begin{array}{c}
\mathbf{a}_{1}^{T}\left(\mathbf{r}_{k}, \mathbf{r}_{s}\right) \\
\vdots \\
\mathbf{a}_{M}^{T}\left(\mathbf{r}_{k}, \mathbf{r}_{s}\right)
\end{array}\right], \\
\mathbf{a}_{i}\left(\mathbf{r}_{k}, \mathbf{r}_{s}\right)= & {\left[\begin{array}{c}
H_{R}^{x} H_{T}^{x} \\
H_{R}^{x} H_{T}^{y}+H_{R}^{y} H_{T}^{x} \\
H_{R}^{x} H_{T}^{z}+H_{R}^{z} H_{T}^{x} \\
H_{R}^{y} H_{T}^{y} \\
H_{R}^{y} H_{T}^{z}+H_{R}^{z} H_{T}^{y} \\
H_{R}^{z} H_{T}^{z}
\end{array}\right], }
\end{aligned}
$$




$$
\mathbf{q}_{k}(t)=\left[\begin{array}{c}
p_{11}(t) \\
p_{12}(t) \\
p_{13}(t) \\
p_{22}(t) \\
p_{23}(t) \\
p_{33}(t)
\end{array}\right],
$$

where $\mathbf{a}_{i}\left(\mathbf{r}_{k}, \mathbf{r}_{s}\right)$ is $6 \times 1$ column vector representing spatial sensitivities of the $i$ th pair of transmitter-receiver to the object located at $\mathbf{r}_{k} \cdot \mathbf{H}_{T}=\left[H_{T}^{x}, H_{T}^{y}, H_{T}^{z}\right]$ and $\mathbf{H}_{R}=\left[H_{R}^{x}, H_{R}^{y}, H_{R}^{z}\right]$ in (4) are the magnetic fields at the object location $\mathbf{r}_{k}$ generated by that transmitter-receiver pair. Equation (3) describes the measurements of an EMI sensor system that are the function of target positions and their polarizabilities as well as a sensing location. The task of EMI sensing of UXO is to recover the principal polarizabilities from measurements for classification.

\section{Method}

Our objective is to estimate model parameters $\mathbf{m}$ that describe targets within view of the sensor and design parameters $\psi$, in particular the location of the sensor. The model parameters $\mathbf{m}$ are the target polarizations and locations. For $\eta$ objects within the sensor field of view, they might be collectively expressed as

$$
\mathbf{m}=\left[\begin{array}{l}
\mathbf{r} \\
\mathbf{q}
\end{array}\right],
$$

where $\mathbf{r}$ contains the location vectors for the $\eta$ objects and q contains their respective polarizabilities. For a single time channel, the dimension of $\mathbf{r}$ is $3 \eta \times 1$ and the dimension of $\mathbf{q}$ is $6 \eta \times 1$. The dimension of $\mathbf{m}$ is $N \times 1$, where $N=3 \eta+6 \eta$. The design parameters $\psi$ related to sensor location are contained in the modeling matrix $A$ of (4). We write (3) as

$$
\mathbf{d}=\mathscr{F}(\mathbf{m}, \psi)+\mathbf{n}(\psi)
$$

where $\mathscr{F}(\mathbf{m}, \psi)$ denotes the nonlinear forward function that maps the $\mathbf{m}$ and $\psi$ on the data. $\mathbf{n}(\psi)$ is the data noise associated with a particular survey design $\psi$. For simplicity, we have suppressed the variable $t$ in (5) and (6).

In standard data processing, the set of experiment design parameters are fixed, and a nonlinear inverse problem is solved for finding model parameters $\mathbf{m}$ [17]. On the other hand, the optimal experiment design problem aims to find the design parameters that are expected to achieve the minimum model uncertainty $[11,12]$. Experiment designs are compared using statistical criteria based on the information matrix. Calculation of the information matrix requires estimating the model covariance matrix. For the nonlinear optimal experiment design problem, we will linearize the forward model function (6) about a reference model. A design criterion based on the linear problem will then be utilized.
3.1. Linearized Model Uncertainty and Optimal Design Criteria. To obtain the analytical expression of a model covariance matrix in the nonlinear inverse problem, we linearize the data functional about a prior estimated model $\mathbf{m}_{0}$ [17]

$$
\mathbf{d} \approx \mathscr{F}\left(\mathbf{m}_{0}, \psi\right)+J \Delta \mathbf{m},
$$

where $\Delta \mathbf{m}=\mathbf{m}-\mathbf{m}_{0}$ and $J$ is the $M \times N$ Jacobian matrix comprising the partial derivatives of the data functional evaluated at $\mathbf{m}_{0}$ for the design $\psi$,

$$
J=\left.\frac{\partial \mathscr{F}}{\partial \mathbf{m}}\right|_{\mathbf{m}_{0}} .
$$

A solution to the linearized equation of (7) can be achieved by minimizing an objective function [17]

$$
\phi=\left\|W_{d}^{-1 / 2}(\Delta \mathbf{d}-J \Delta \mathbf{m})\right\|^{2}+\left\|C_{0}^{-1 / 2} \Delta \mathbf{m}\right\|^{2},
$$

where $\Delta \mathbf{d}=\mathbf{d}-\mathscr{F}\left(\mathbf{m}_{0}, \psi\right)$ are the data residuals between the measured data and the data predicted at the model $\mathbf{m}_{0} . W_{d}$ is generally treated as a data covariance matrix and $C_{0}$ is a prior model covariance matrix. The first term in (9) is the data misfit, measuring the discrepancies between the observed and theoretical quantities. The second term is a damping or regularization term that plays a role in controlling the size of a solution model relative to model $\mathbf{m}_{0}$. The formal linearized least square solution for (9) is given by [17]

$$
\begin{aligned}
\mathbf{m} \approx & \mathbf{m}_{0} \\
& +\left(J^{T} W_{d}^{-1} J+C_{0}^{-1}\right)^{-1} J^{T} W_{d}^{-1}\left(\mathbf{d}-\mathscr{F}\left(\mathbf{m}_{0}, \psi\right)\right) .
\end{aligned}
$$

The corresponding posterior covariance matrix $C_{m}$ can be approximated as [17]

$$
C_{m} \approx\left(J^{T} W_{d}^{-1} J+C_{0}^{-1}\right)^{-1} .
$$

As indicated in $[13,17]$, a model $\mathbf{m}_{0}$ used for the linearization is often obtained as a local optimum point through an iterative optimization algorithm. The posterior $C_{m}$ of (11) estimated at convergence is viewed as a linearized measure of uncertainty in the estimated model parameters. This estimate forms the basis for various experimental design approaches $[11,12]$.

In the standard experimental design approaches, the model covariance matrix is converted into some scalar functions such as its determinant or trace, that is, $\Phi_{D}=$ $\left|C_{m}\right|$ or $\Phi_{A}=\operatorname{tr} C_{m}$. Then an optimal design $\psi$ might be sought in terms of the $D$ - and $A$-criteria [11, 12], given by $\min _{\psi} \Phi_{D}$ and $\min _{\psi} \Phi_{A}$, respectively. Under assumption that the estimates are multivariate Gaussian, $C_{m}$ in the model space might be pictured as an ellipsoidal confidence region about the parameter estimates. Geometrically, $\Phi_{D}$ or $\Phi_{A}$ measures the size of this ellipsoid either in terms of its volume or its average semiaxes lengths. The smaller the ellipsoidal region, the smaller the uncertainty in parameter estimates. Posing $\Phi_{D}$ or $\Phi_{A}$ as a function of experimental parameters $\psi$ makes it possible to seek an optimal experiment over the 
experiment space and render the minimal postexperimental uncertainty in model estimates.

The optimization of experimental designs $\psi$ in our case is conditional on the assumed model $\mathbf{m}_{0}$. In principle, the optimal design can be achieved only when the prior estimate $\mathbf{m}_{0}$ is close to the true parameters. In reality, such an assumption might not always hold. Thus for a nonlinear model, it is necessary to have a procedure in which the design can be sequentially updated upon the improved estimate of the model parameters after each experiment. In the following we present a $D$-criterion-based sequential experimental design.

3.2. Sequential Experimental Design Optimization. In this section, we follow the approaches of augmenting experimental data $[11,13,14]$ to obtain a sequential experimental design criterion and procedure in terms of the posterior covariance matrix.

Starting a current survey stage denoted as $s$, we wish to find or predict the next sounding, denoted as future stage $s+1$. The sensitivity matrix at stage $s+1$ can be expressed as

$$
B_{s+1}=\left[\begin{array}{c}
B_{s} \\
J_{s+1}
\end{array}\right]
$$

where $B_{s}^{T}=\left[J_{1}^{T}, \ldots, J_{s}^{T}\right]$ is a composite of the sensitivity matrices for $s$ experiments at stage $s$ and $J_{s+1}$ represents a sensitivity matrix that is to be determined via optimizing the criterion below. $B_{s+1}$ is formed by appending a future $J_{s+1}$ to the existing $B_{s}[11,13,14]$. This is equivalent to adding data from a new sounding. Denote the model covariance matrix at stage $s$ as $C_{s}$. With (11), its inverse can be expressed as

$$
C_{s}^{-1}=\left(B_{s}^{T} W_{s}^{-1} B_{s}+C_{0}^{-1}\right)
$$

where $W_{s}$ is the data covariance matrix at the $s$ th stage, given by

$$
W_{s}=\left(\begin{array}{ccc}
\Sigma_{1} & 0 & 0 \\
0 & \ddots & 0 \\
0 & 0 & \Sigma_{s}
\end{array}\right),
$$

where $\Sigma_{s}$ is a block data covariance matrix of size $M \times M$ at the $s$ th sounding. For stage $s+1$, the posterior covariance matrix is given by

$$
C_{s+1}^{-1}=\left(B_{s+1}^{T} W_{s+1}^{-1} B_{s+1}+C_{0}^{-1}\right) .
$$

Inserting (12) and using (13), the inverse posterior covariance matrix of (15) at step $s+1$ may be rewritten as

$$
\begin{aligned}
C_{s+1}^{-1} & =\left(B_{s}^{T} W_{s}^{-1} B_{s}+C_{0}^{-1}+J_{s+1}^{T} \Sigma_{s+1}^{-1} J_{s+1}\right) \\
& =\left(C_{s}^{-1}+J_{s+1}^{T} \Sigma_{s+1}^{-1} J_{s+1}\right) .
\end{aligned}
$$

Using the matrix determinant lemma [18], we can have

$$
\begin{aligned}
\left|C_{s+1}^{-1}\right| & =\left|C_{s}^{-1}+J_{s+1}^{T} \Sigma_{s+1}^{-1} J_{s+1}\right| \\
& =\left|C_{s}^{-1}\right|\left|I_{M}+\widetilde{J}_{s+1} C_{s} \widetilde{J}_{s+1}^{T}\right|,
\end{aligned}
$$

where $I_{M}$ is an identity matrix with the size of $M \times M$ and $\tilde{J}_{s+1}=\Sigma_{s+1}^{-1 / 2} J_{s+1}$. Therefore, we can introduce a new function as

$$
\mathscr{I}_{D}=\ln \frac{\left|C_{s+1}^{-1}\right|}{\left|C_{s}^{-1}\right|}=\ln \frac{\left|C_{s}\right|}{\left|C_{s+1}\right|}=\ln \left|I_{M}+\widetilde{J}_{s+1} C_{s} \widetilde{J}_{s+1}^{T}\right| .
$$

Equation (18) is a scalar measure of the model covariance reduction that can be obtained by adding a new sounding to the previous $s$ soundings. Conversely, $\mathscr{I}_{D}$ may be called an expected information gain. The core part in the objective function $\mathscr{I}_{D}$ is $\widetilde{J}_{s+1} C_{s} \widetilde{J}_{s+1}^{T}$ that is a model covariance matrix $C_{s}$-weighted product of the scaled sensitivity matrix $\widetilde{J}_{s+1}$ at the candidate sensing location. The way (18) works may be understood qualitatively. If the elements of model covariance matrix are larger at previous $s$ stages, $\mathscr{I}_{D}$ will tend to be large by seeking a new experiment that can increase sensitivities in $\widetilde{J}_{s+1}$ and thus reduce the model uncertainty expressed in (15). On the other hand, if the elements of model covariance matrix are already smaller at the previous $s$ stages at which large sensitivities were built upon, the value of $\mathscr{I}_{D}$ would be small when adding a new experiment that likely resembles one of the previous ones.

The above presentation is general and can be reduced to some specific case. The form of (18) is similar to those in $[13,14]$, but its inclusion of the covariance matrices makes it convenient to incorporate available a priori knowledge about the model and accommodate various types of measurement noise into an optimal experimental design process. Specifying the prior covariance matrices requires statistical noise models that are representative of the experiment. However such statistical noise models are not always known or easily defined in reality. To simplify, one common practice is to ignore $C_{0}$ and assume a uniform uncorrelated data noise $[13,14]$. In our current implementation, we also simply choose $C_{0}^{-1}=0$ which represent no prior information about model in the beginning. However in the sequel, this choice is automatically updated as shown in (16) where $C_{s}$ from the previous survey locations can be viewed as prior model information for a next intended survey location and is embedded in (18). For a data covariance matrix, we consider an uncorrelated nonuniform error distribution in this study. That is, a standard error is assigned as a summation of the baseline error $\delta_{j}$ and a percentage $\vartheta$ of an observed datum [19]. Then the diagonal entry of $W_{i i}$ is written as $W_{i i}=\left(\delta_{i}+\vartheta|d|_{\mathrm{obs}, i}\right)^{2}$ for $i$ th datum.

Having obtained the $D$-optimality function (18), we can implement a sequential experimental design (SED) for sensor placement in the following manner.

Step 1. Given the survey parameters as $\psi_{s}$ at the sth stage, finding the best model parameters $\mathbf{m}$ is stated as the minimization of a nonlinear least squares function with some constraints (for algorithm details see $[10,20,21]$ )

$$
\begin{aligned}
\widehat{\mathbf{m}}_{s} & =\arg \min _{\mathbf{m}}\left\{\left\|W_{s}^{-1 / 2}\left(\mathbf{d}-\mathscr{F}\left(\mathbf{m}, \psi_{s}\right)\right)\right\|^{2}: \mathbf{m}\right. \\
& \left.=(\mathbf{r}, \mathbf{q}), \quad\|\Delta \mathbf{r}\| \leq \Delta_{r}, \mathbf{q} \in S_{+}\right\},
\end{aligned}
$$




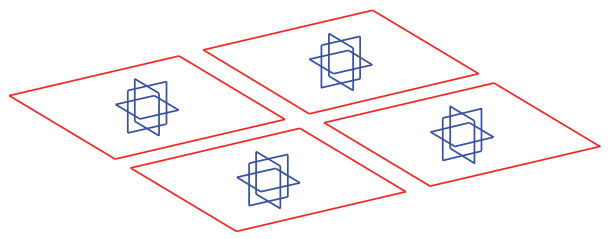

(a)

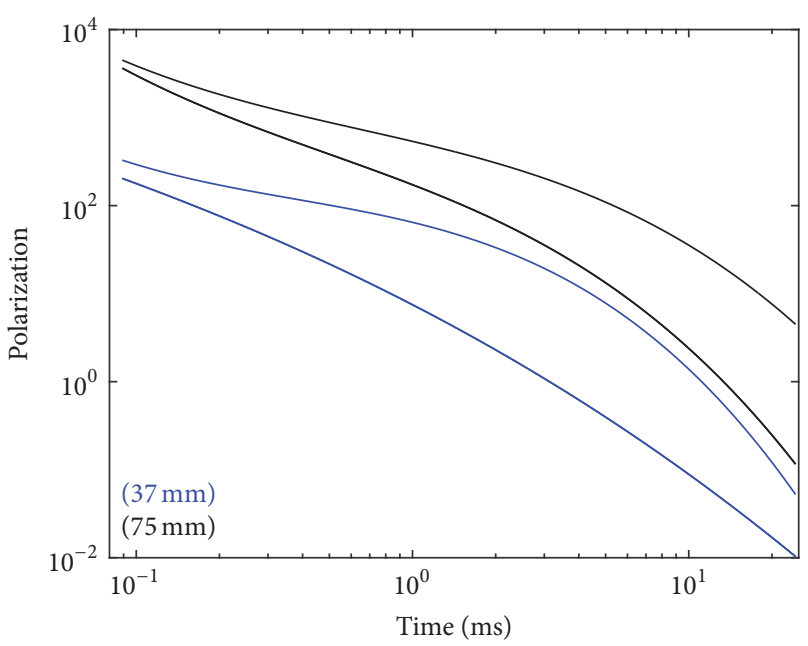

(b)

FiguRE 1: (a) TEMTADS2x2: a multistatic system consisting of 4 coplanar transmitters and 4 triaxial receiver cubes. Each transmitter is $40 \mathrm{~cm}$ $\times 40 \mathrm{~cm}$ and each receiver $8 \mathrm{~cm} \times 8 \mathrm{~cm}$. (b) Two sets of polarizations used for the numerical experiments. The blue and black solid curves represent polarizations decays of $37 \mathrm{~mm}$ and $75 \mathrm{~mm}$ projectiles.

where $\Delta_{r}$ is a positive scalar used to provide a local trust region within which $\Delta \mathbf{r}$ is allowed to change and $\mathbf{q} \in S_{+}$ represents that $\mathbf{q}$ is constrained to be the elements of the set $S_{+}$of symmetric positive semidefinite matrices.

Step 2. Given the current model $\widehat{\mathbf{m}}_{s}$ and starting from the most recent sensor position in $\psi_{s}$, we predict next sensing location represented as $\psi_{s+1}$ by maximizing

$$
\begin{aligned}
\psi_{s+1} & =\arg \max _{\psi} \mathscr{I}_{D}\left(\widehat{\mathbf{m}}_{s}, \psi_{s}, \psi\right) \\
& =\arg \max _{\psi} \ln \left|I_{M}+\widetilde{J}(\psi) C_{s} \widetilde{J}^{T}(\psi)\right| .
\end{aligned}
$$

The optimization can be carried out via the Matlab [22] function fmincon.

Repeat this survey augmenting process until a stopping criterion is attained. This stopping criterion may be a predefined threshold of information gain or a maximum number of sensing stages.

\section{Results}

In this section, we evaluate the SED with two portable EMI sensor systems: one is the TEMTADS2x2 (Transient Electromagnetic Multisensor Towed Array Detection System) [23]; the other is the MPV (Man Portable Vector sensor) [24, 25]. Each sensor is described in the related subsections.

4.1. Synthetic Experiments with TEMTADS2x2. First, we present results with synthetic TEMTADS2x2 time-domain data with a noise level of $7 \%$ to illustrate the capability of the optimal experimental design for the sensor placement.

TEMTADS2x 2 is a sensor system that consists of 4 coplanar small transmitters and 4 sets of tri-axial receiver cubes [23] (Figure 1(a)). The sizes of each transmitter and receiver are $40 \mathrm{~cm} \times 40 \mathrm{~cm}$ and $8 \mathrm{~cm} \times 8 \mathrm{~cm}$. Two objects, $37 \mathrm{~mm}$ and $75 \mathrm{~mm}$ projectiles, are used in the numerical experiments. Their polarizabilities are shown in Figure 1(b).

In the first experiment, we consider a scenario where the two objects are initially sensed near the edge of the instrument. The locations of the two objects are $\mathbf{r}_{1}=$ $(0.20,0.30,-0.15) \mathrm{m}, \mathbf{r}_{2}=(0.10,0.15,-0.35) \mathrm{m}$. Figure $2(\mathrm{a})$ shows the relative position in the first place between the sensor footprint (the black dashed square) and the object locations (the two red circles). We inverted the initial sounding data and obtained the estimated locations of the two objects: $\widetilde{\mathbf{r}}_{1}=(0.17,0.36,-0.15) \mathrm{m}, \widetilde{\mathbf{r}}_{2}=(0.11,0.10,-0.24) \mathrm{m}$. Figure 2(b) shows that the recovered polarizabilities (the solid curves) are fluctuated and far from the true ones (dashed curves).

Given current model information extracted from the initial sounding data, we ran the SED to predict the next sounding location. The expected information gain is 98.10 if moving sensor from previous sounding 1 to predicted sounding 2. At the suggested place (green cross) in Figure 2(a), the sensor (the green square) would center on top of the two objects. Following the SED forecast, we took the second sounding and inverted the combined data from the two soundings. As a result (Figure 2(c)), we obtained significant improvements over the estimated locations of the two objects and the recovered polarizabilities. As an experiment, we continued to run the SED for a next sounding given the previous measurements. Relative to first two soundings, the SED predicted that the expected information gain would be 14.42 at another new sounding, shown as a blue square in Figure 2(a). Again taking the measurements in this newly intended sensor location from the SED and inverting all three sounding data simultaneously, we further achieved some improvements in the recovery of polarizabilities of $37 \mathrm{~mm}$ projectile. Subsequently, we would have a small 


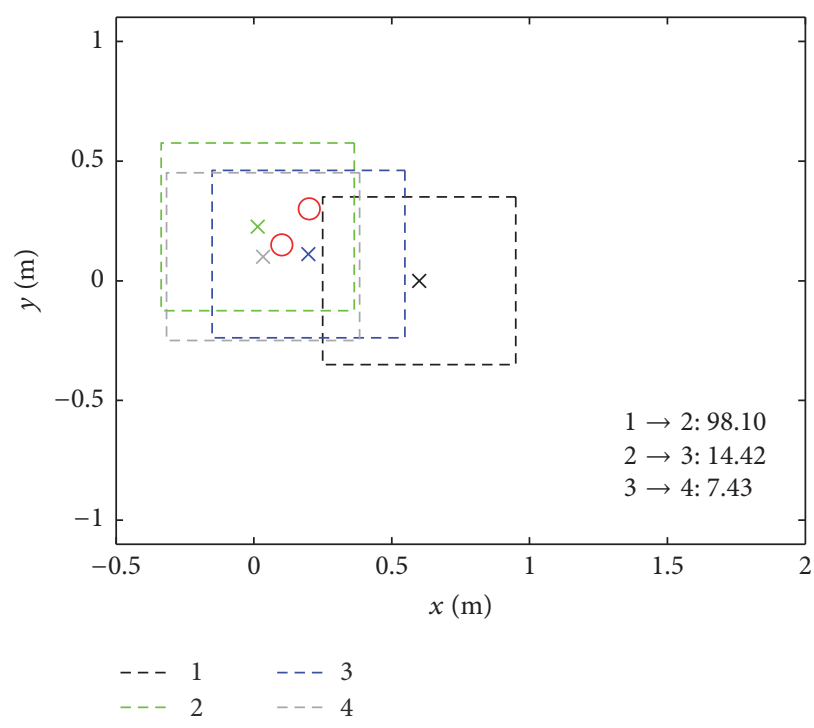

(a)

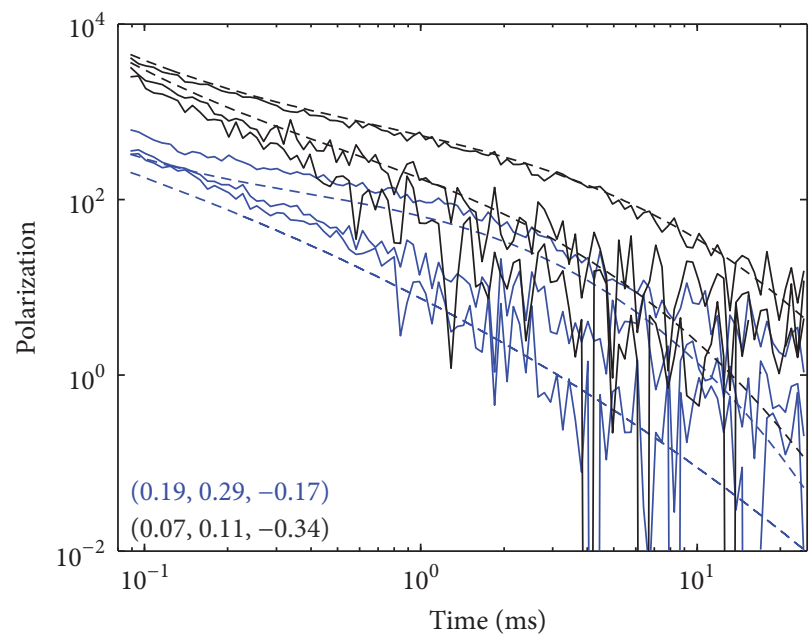

(c)

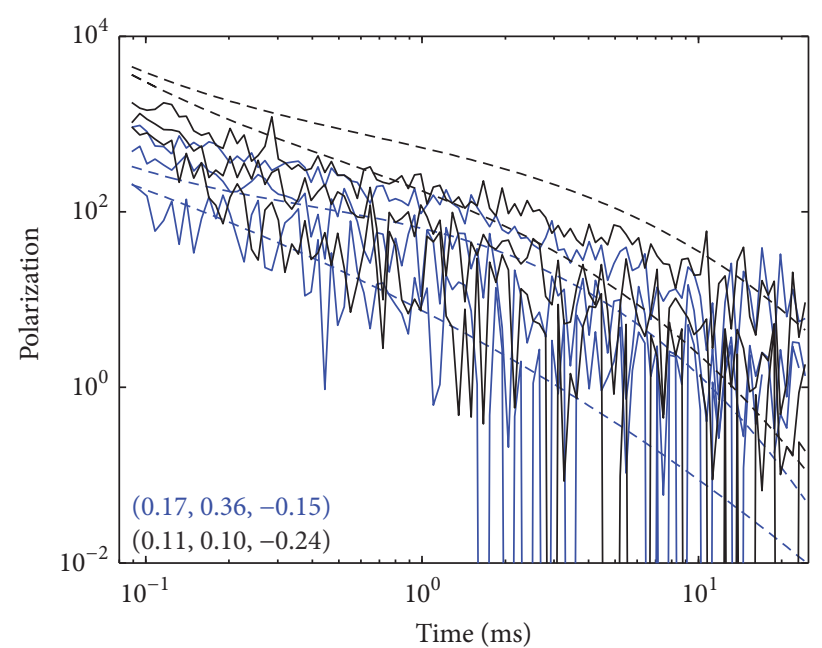

(b)

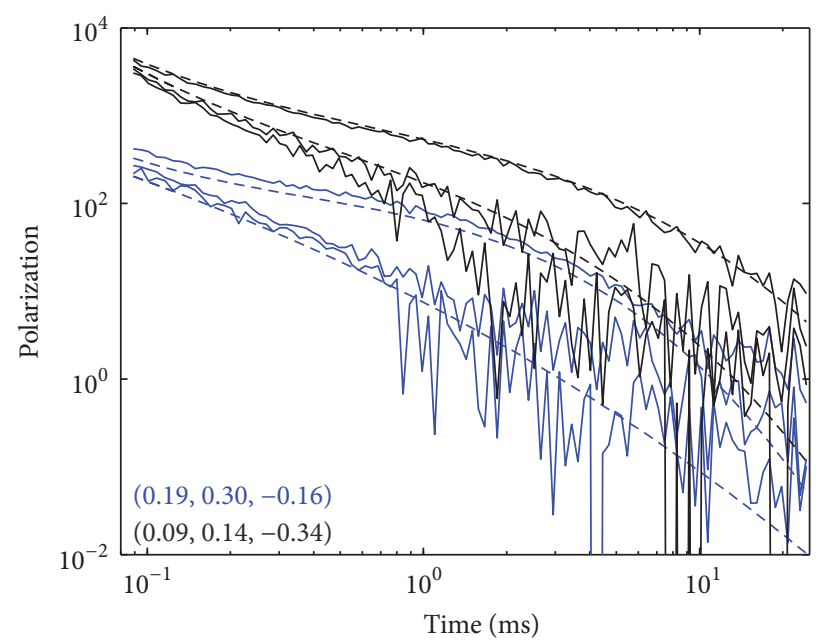

(d)

FIGURE 2: Experiment 1: two objects near the sensor edge. (a) Two red circles represent the true locations of the objects. The black square delineates the footprint for the initial sensor location and the other color squares indicate the predicted positions. (b) The recovered polarizabilities from the initial sounding (s1) data. (c) The recovered polarizabilities from the sounding data [s1 s2]. (d) The recovered polarizabilities from the sounding data [s1 s2 s3]. In the polarization subplots, the solid curves represent the recovered polarizabilities and the dashed ones the true ones.

information gain of 7.43 for a 4 th future sounding relative to the previous three soundings. Given decreasing information gain gradually from the SED, we stopped taking any more measurements.

In the second experiment, we mimic another real case where both objects are close to the sensor edges but one is inside the sensor footprint and the other one is outside of it. The locations of the objects are at $\mathbf{r}_{1}=(0.45,-0.21,-0.15) \mathrm{m}$ and $\mathbf{r}_{2}=(0.10,0.15,-0.35) \mathrm{m}$. Figure $3(\mathrm{a})$ shows the relative position initially between the sensor footprint (the black dashed square) and the object locations (the two red circles). Inverting the first sounding data derives the locations of the two objects: $\widetilde{\mathbf{r}}_{1}=(0.44,-0.21,-0.14) \mathrm{m}, \widetilde{\mathbf{r}}_{2}=(0.17,0.12$, $-0.36) \mathrm{m}$. For the $37 \mathrm{~mm}$ projectile, its recovered polarizabilities (in the blue solid curves) are acceptable although the secondary polarizabilities show some fluctuation in late times (Figure 3(b)). But for $75 \mathrm{~mm}$ projectile that is outside of sensor footprint, the recovered polarizabilities are noisy. However the situation improves after applying the SED method. For example, the suggested new sounding (the green square) in Figure 3(a) would cover both objects within sensor footprint. The corresponding expected information gain is 31.23 assuming measurements would be taken in this place. By taking the measurements as suggested by the SED and then inverting composite data from both soundings, we not only obtain the cleaner polarizabilities of the $37 \mathrm{~mm}$ projectile but also well recover the polarizabilities of the $75 \mathrm{~mm}$ projectile (Figure 3(c)). After that, we might want to know what the SED can continuously forecast. Relative to the current measurements, the expected information gain would 


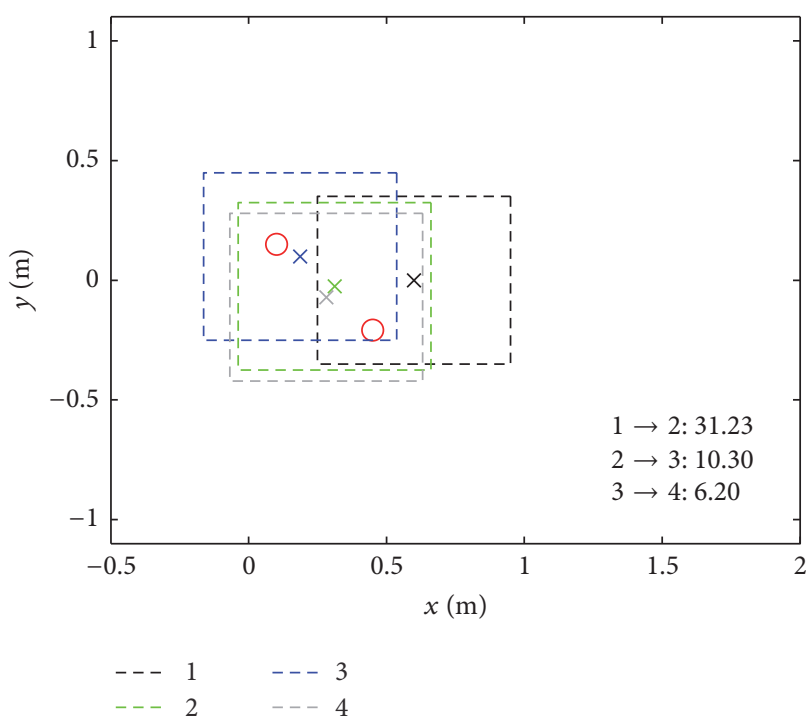

(a)

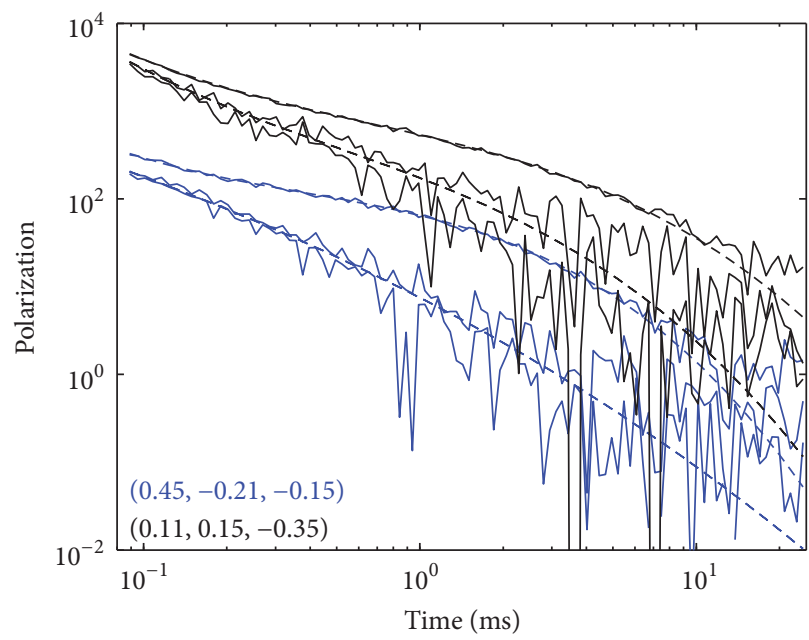

(c)

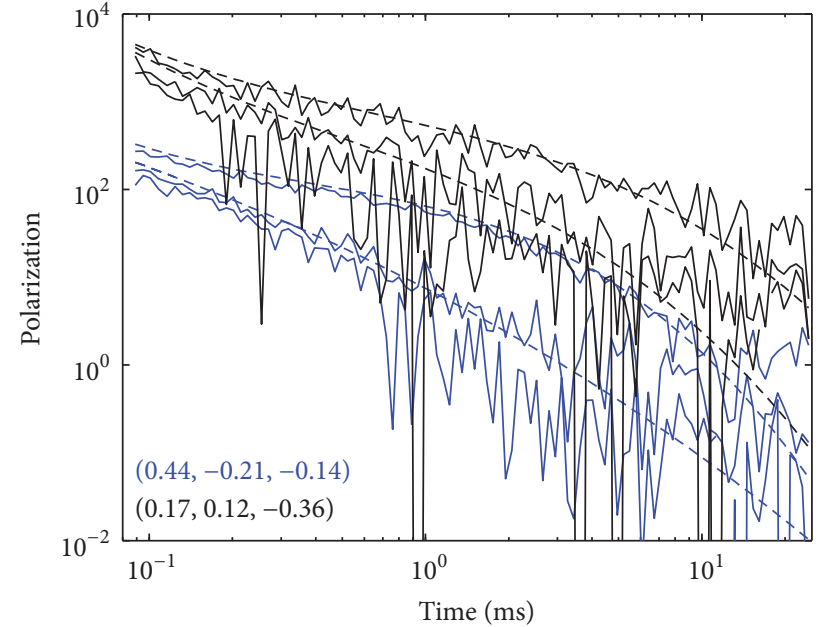

(b)

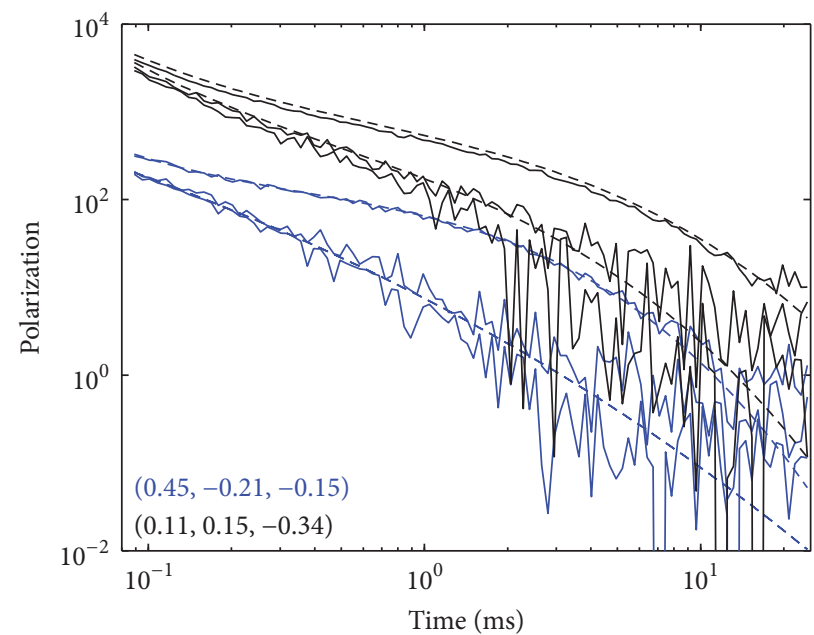

(d)

Figure 3: Experiment 2: two objects close to sensor edges. Caption is the same as that in Figure 2.

be 10.30 for the next sounding. Taking the measurements at the suggested 3rd sounding location, we further pin down the fluctuation of the recovered polarizabilities in the late times. Similar to the first experiment, we ran the SED to examine a possible fourth sounding and observe that only the small information gain of 6.2 can be achieved. We decided not to acquire additional data with the suggested sounding.

From the above experiments, we see that the SED predicts to place a sensor toward the locations of objects. Recall that EMI sensing is quasistatic and signals fall off rapidly about $1 / r^{6}$, where $r$ is the distance from a sensor to an object. By reducing that distance in an optimal way, the SED attempts to increase sensing sensitivities and the SNR as well. This is consistent with the physical nature of the problem. As shown in Figure 4, the information gain in (18) as a function of sensing location can achieve maximum values around the objects' locations. This concave property justifies the use of the $D$ optimality-based optimal experimental design to forecast an optimal sensor placement.

The previous studies on inversions of multiple objects [8-10] have shown that a single-object model may lead to the adverse consequence of missing targets of interest and is often not sufficient to guarantee an accurate recovery of target polarizabilities. Similarly in the SED test when assuming a single-object model for the above two-object examples, we obtained incorrect results: missing recovery of one anomaly or both (not shown here). Next, we are interested to establish whether the SED is effective for an overrepresented model, that is, assuming the number of objects larger than the actual one.

In the third experiment, we inspect the SED result given a overestimated number of objects. Consider a single object (37 mm projectile) at $\mathbf{r}=(0.10,0.15,-0.35) \mathrm{m}$. The object is outside of the sensor footprint. Figure 5(a) shows the initial sensing position relative to the object. In the 


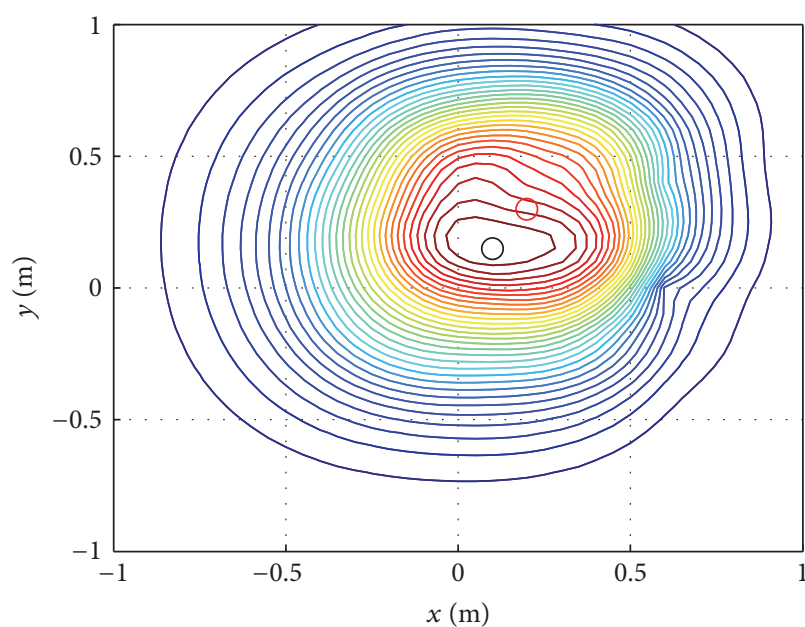

(a)

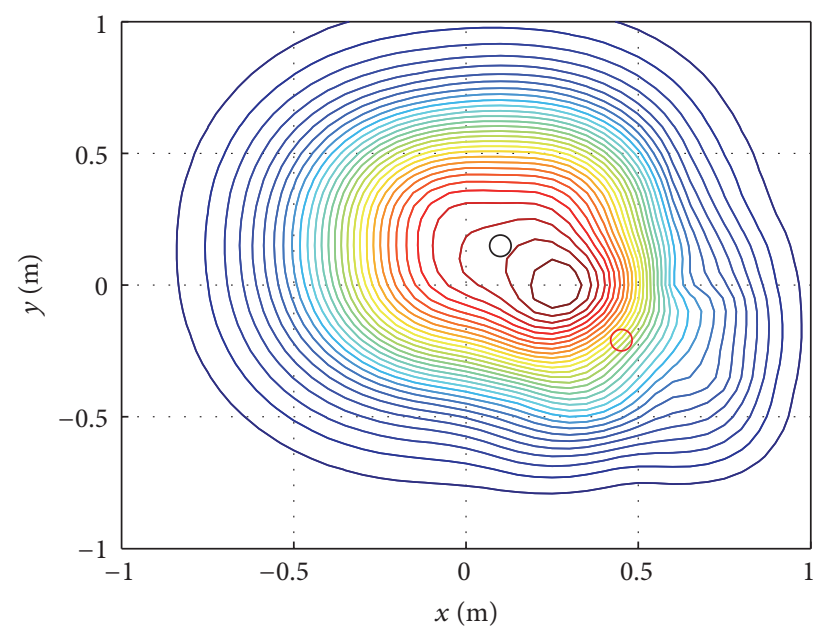

(b)

FIGURE 4: Map of Information gain as a function of sensor locations: (a) Experiment 1; (b) Experiment 2. The two circles denote the locations of the two objects.

following, we simply suppose a two-object model. Given the initial sounding data that were collected over a singleobject anomaly, we inverted them as if there were two objects. Figure 5(b) shows the recovered polarizabilities in the first location. The black jittery curves correspond to a false source at $\widetilde{\mathbf{r}}_{1}=(-0.33,0.10,-0.36) \mathrm{m}$. For the actual object (blue solid curves), its primary polarizability decay curve is somehow recovered but its amplitude is smaller than the true one (dashed curves). The secondary polarizabilities are noisy. The estimated location of the true object is given by $\widetilde{\mathbf{r}}_{2}=(0.13,0.17,-0.30) \mathrm{m}$. Given the current inaccurate information extracted from the initial sounding data, the SED predicts a new sensing place (the position of the green square in Figure 5(a)) with the expected information gain of 128.12. Conducting the measurements as the SED forecasted and performing the inversion with the two-sounding data, we immediately get the recovered polarizabilities that match well with the true ones (Figure 5(c)). Meanwhile, the false source is inverted now as being outside of the current sounding range and the associated polarizabilities are with very small amplitudes. The third SED predicted sounding (the blue square in Figure 5(a)) suggests a place covering the spurious source inverted in the current model parameters. The expected information gain is achieved as 12.77 with respect to the two previous real soundings. Assume that we continued to take the measurements according to the SED prediction. The results of inverting all three sounding data are quite similar to those of inverting the two sets of data (Figures 5(c) and 5(d)). After the three soundings, the fourth theoretic sensing location is directly over $37 \mathrm{~mm}$ projectile. However, with the relative small information gain of 6.73 , it is unnecessary to acquire more data.

This experiment shows that an overrepresentation model in the SED might be a simple, feasible way to deal with the unknown number of objects.
4.2. Experiments with the MPV. To further evaluate the SED, we conducted experiments with the MPV [24, 25]. In this development stage, we considered an indoor environment for an operational matter of efficiency since it needs a number of efforts of tuning and modifying the SED program when transiting from a numerical setup to the real-time processing.

Figure 6(a) schematically shows the MPV [24, 25]: a multistatic sensor system that consists of three orthogonal transmitters: a $z$-directed circular one with the diameter of $50 \mathrm{~cm}$ and $x$ - and $y$-directed rectangular ones with the sizes of $54 \mathrm{~cm} \times 54 \mathrm{~cm}$ and $54 \mathrm{~cm} \times 50 \mathrm{~cm}$, respectively. Five sets of triaxial receiver cubes (each $10 \mathrm{~cm} \times 10 \mathrm{~cm}$ ) are distributed on the circular transmitter plane. Referring to Figure 6(b), the sensor in the setup would be moved manually on the wood plate to a specified mark and targets were positioned below the plate for a desired configuration. Experimental objects include small, medium, and large ISOs (Industrial Standard Object) (Figure 6(c)). Note that the experiments were taken in the Black Tusk Geophysics office in the day time where noise was strong probably due to various nearby inside and outside electronic interferences. These environment factors might introduce correlated noise. That noise was compensated by taking a background measurement in the absence of the test items before each experiment and by subsequently subtracting background contribution from the data. Considering that the method would be deployed on a real-time system, we assumed an uncorrelated nonuniform noise model. As shown below the SED performance, the assumption appears reasonable.

As mentioned previously, we attempted to simulate multiple-target cases that might be encountered in real scenarios: for example, there might be one or two targets that are near or outside the footprint of the instrument. The goal of the experiments is to evaluate how the SED would guide to characterize targets near the instrument edges by taking an additional data sounding. We presented two SED experiments with twoobject and three-object cases, respectively. 


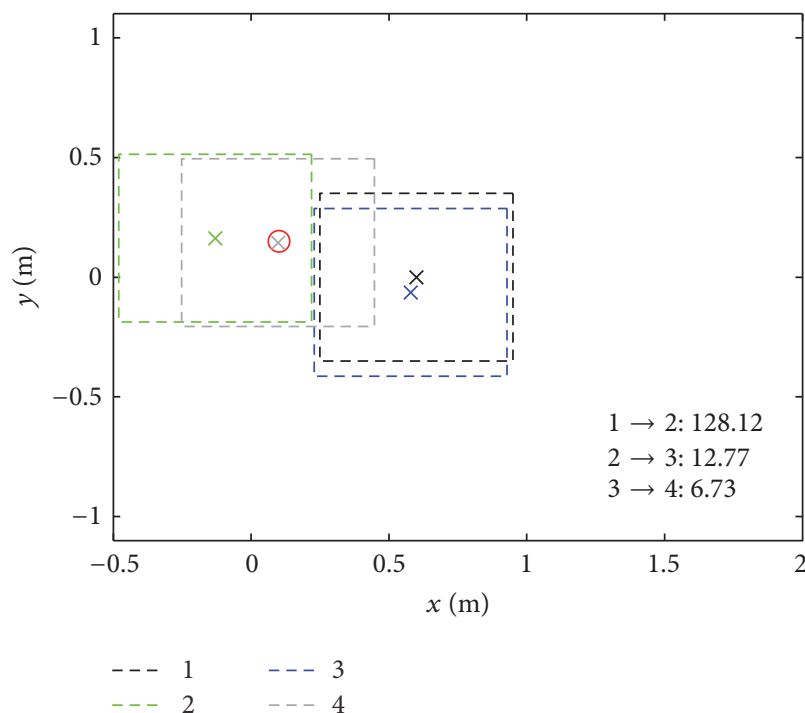

(a)

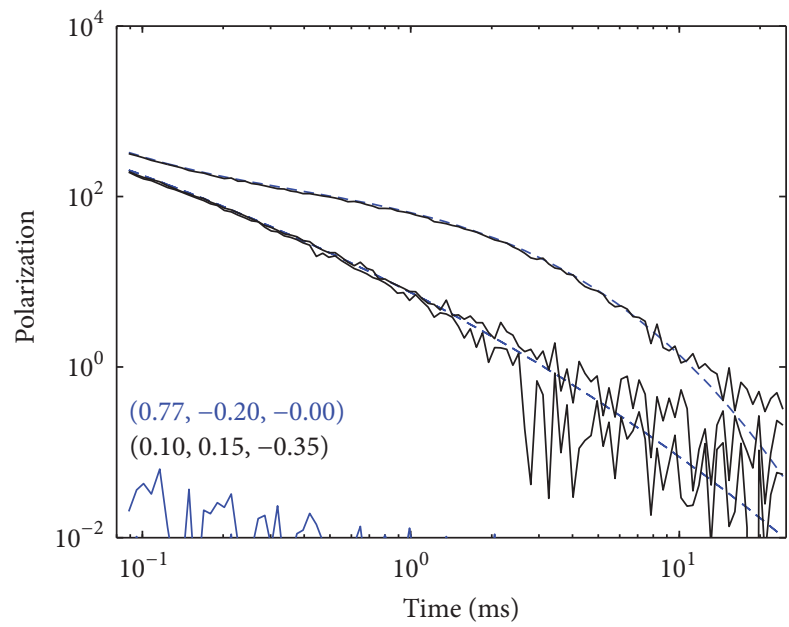

(c)

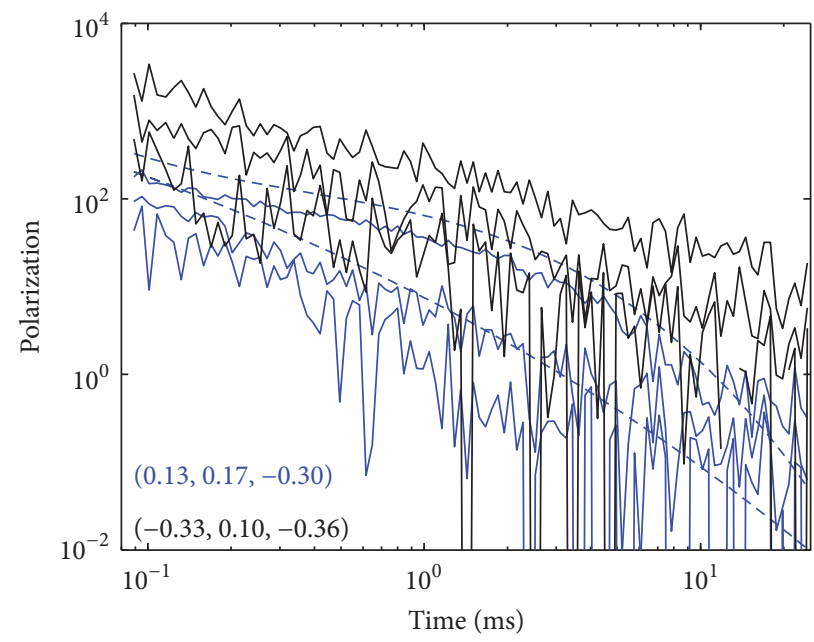

(b)

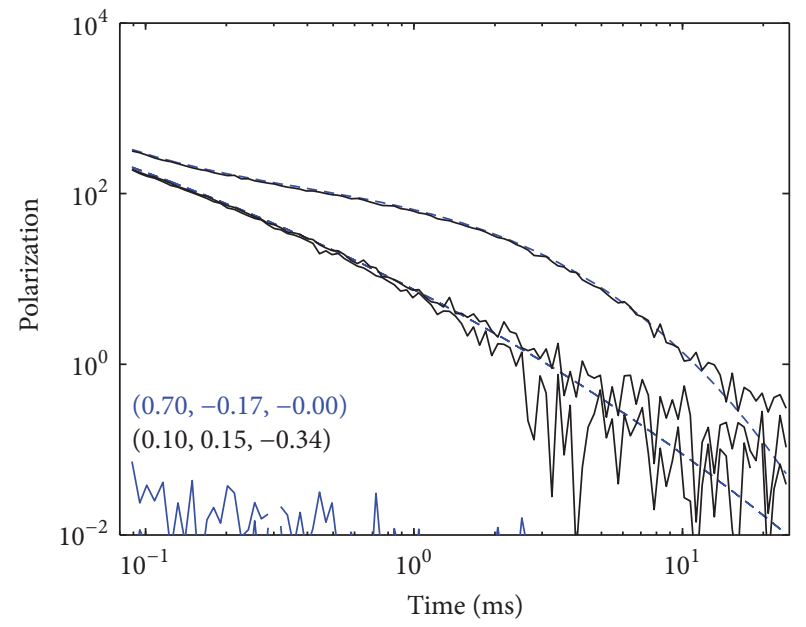

(d)

FIGURE 5: Experiment 3: single-object close to sensor edges. Caption is the same as that in Figure 2.

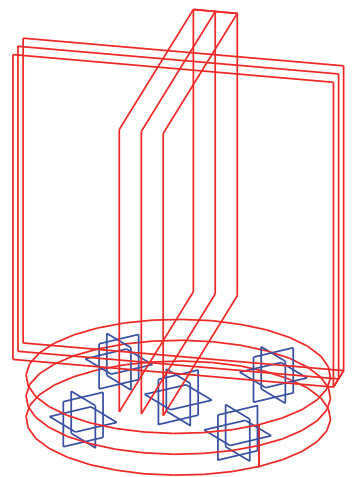

(a)

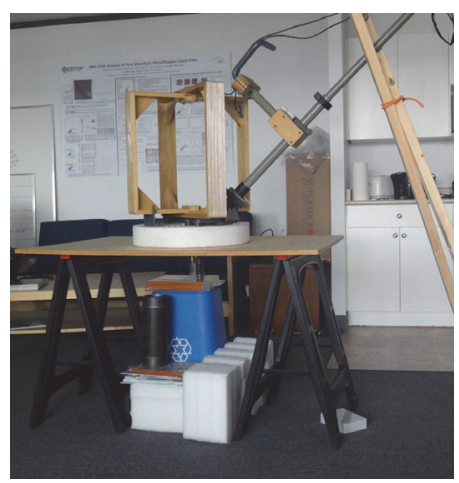

(b)

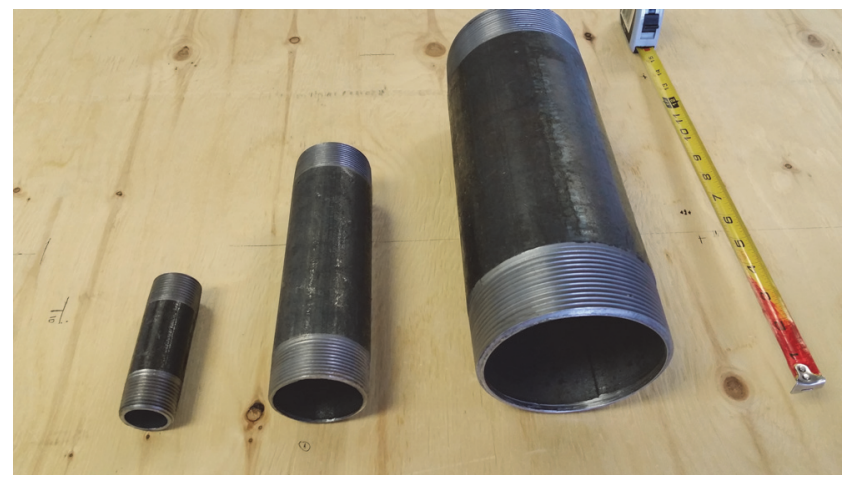

(c)

FiguRE 6: (a) MPV: a multistatic system consisting of a $z$-directed circular transmitter with the diameter of $50 \mathrm{~cm}$ and $x$ - and $y$-directed rectangular transmitters with size of $54 \mathrm{~cm} \times 54 \mathrm{~cm}$. 5 triaxial receiver cubes (each $8 \mathrm{~cm} \times 8 \mathrm{~cm}$ ) are distributed on the circular transmitter plane. (b) A snapshot of the indoor experiment setup. (c) Photos of small, medium, and large ISOs used in the experiments. 


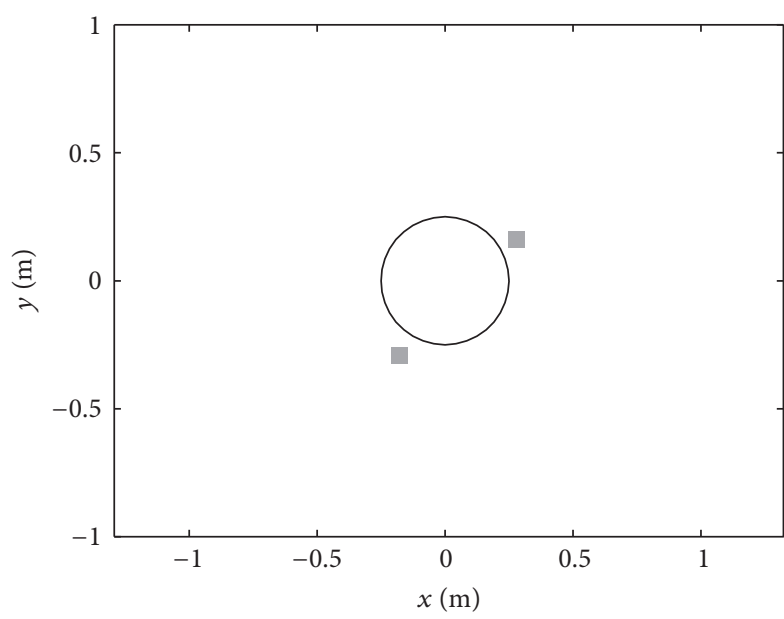

(a)

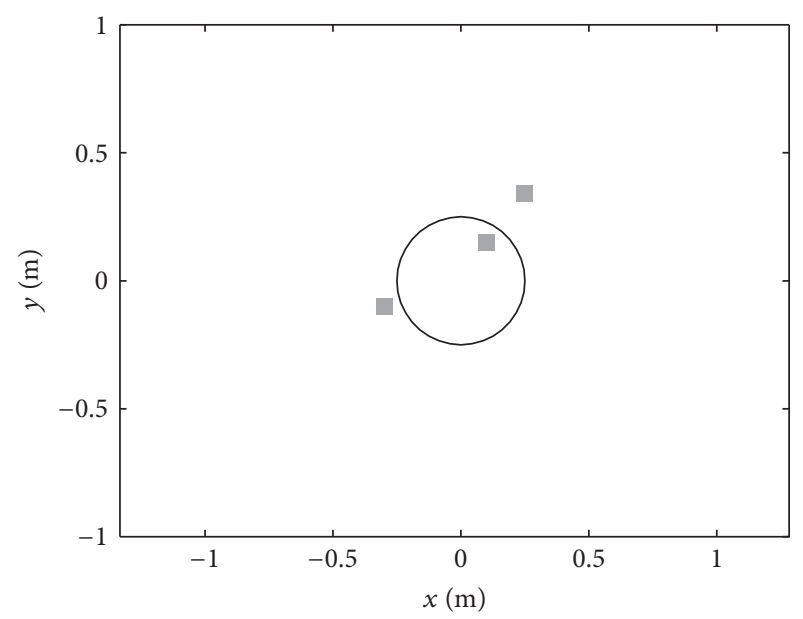

(b)

FIgure 7: Target locations and initial sensor footprint in the SED experiment setup with the MPV. (a) Two-object example. (b) Three-object example. In the initial sounding (S1), a black circle denotes the sensor footprint and gray squares the true locations of the objects.

In the two-object case shown in Figure 7(a), there are small and large ISOs (denoted by gray squares) that were positioned at the opposite sides of the sensor footprint (black circle). Inverting the initial sounding data (S1) provided a rough estimation of target locations, denoted by the small circles in Figure 8(a). The recovered polarizabilities are presented in Figures 8(b) and 8(c), with the dashed gray curves showing the reference polarizabilities of the ISOs. Overall, the primary polarizabilities of both targets were fairly recovered with some level of jitter at late times. However the recovered secondary and tertiary polarizabilities for both small and large ISOs appear noisy (Figures 8(b) and 8(c)). Given the model parameters derived from the initial sounding (S1), the SED predicted the second sounding at $(0.28,0.32) \mathrm{m}$ with a large expected information gain of 83.75 . It suggested to position the instrument over the inverted small target. Shown as a green circle in Figure 8(d), we moved the sensor to the second sounding location as the SED guided and acquired the additional data. The inversion with the additional sounding seems to mainly affect recovery of the small object. Its location has some adjustments relative to that derived from the initial sounding inversion. Its polarizabilities become smooth in Figure 8(e). Under the updated model parameters, the SED suggested a third sounding at $(-0.14,-0.28) \mathrm{m}$ with information gain of 23.39. We followed the SED to move the sensor in the new position (the big blue circle in Figure $8(\mathrm{~g})$ ) that covers the deep and large target. By inverting all three sets of data, we obtained improvements on the recovered polarizabilities of the small and large targets, which became smoother and cleaner. One possible advantage of the SED is that it is able to provide some knowledge about the future state but without a need of real action. For instance after we took three shots of measurements, the running of the SED points out a fourth sounding at $(-0.22,-0.16) \mathrm{m}$, which is a small offset relative to the third sounding location. And the expected information gain is 8.12 for the new sounding, which is small as compared to previously taking second and third soundings. Thus in this example, we can stop collecting data for either reaching a predefined number of sounding or having a small expected information gain.

In the three-object case shown in Figure 7(b), a medium ISO was inside of the sensor footprint and a small and a large ISO were both outside of the sensor footprint. Data acquired at the initial stage (S1) were initially inverted by assuming a two-object model. The polarizabilities of the medium and large ISOs were well inverted and their recoveries appear not affected by the presence of outside small ISO. The signal contribution of the small ISO to the data S1 is negligible relative to the two other objects. This was verified by inverting the data S1 for a three-object model. Results in Figures 9(d)9(f) show inversion of the initial sounding data does not render a small ISO-like object, rather an additional, false medium ISO-like object (Figure 9(d)). In other words, the use of the single sounding data is not sufficient to accurately resolve the three targets in this configuration. In practice, we conducted the SED using the results of the two-object inversion. The SED suggested moving the sensor to a position of $(0.11,0.19) \mathrm{m}$ to cover the upper-right target in the current inversion result. The expected information gain was 28.46 for the next possible sounding. We took the second measurement and inverted the two sets of data simultaneously with a twoobject model. In Figure 9(g), the big green circle denotes the new sensor location and the two small circles the inverted locations of the two objects. In the updated inversion, the polarizabilities of the medium ISO were recovered with some overshoot relative to the reference ones. However unlike the inversion in S1 stage, we did not see the polarizabilities matching the large ISO. Instead, we obtained one different, new set of recovered polarizabilities that agree with those of the small ISO. This suggested that an additional target was present, distinct from the two targets found at S1. This motivated the use of a three-target inversion. Using a 3object model and inverting the two sets of sounding data, we were able to resolve all three targets (their locations and polarizabilities) in a noisy setting (Figures 9(j)-9(1)). We stopped collecting data after reaching the sounding number 


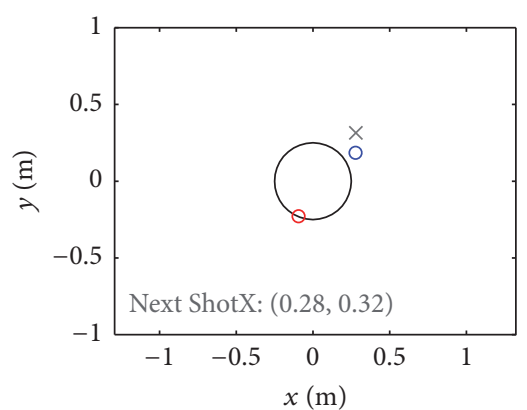

$-\mathrm{S} 1$

(a)

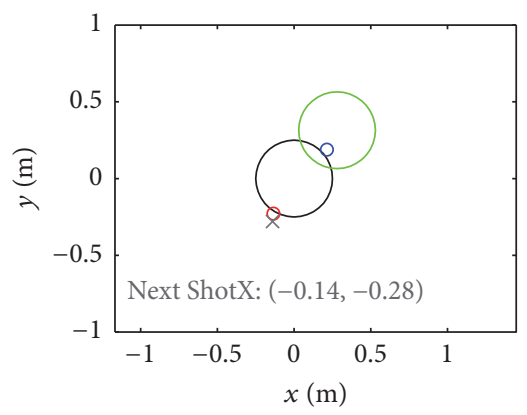

(d)

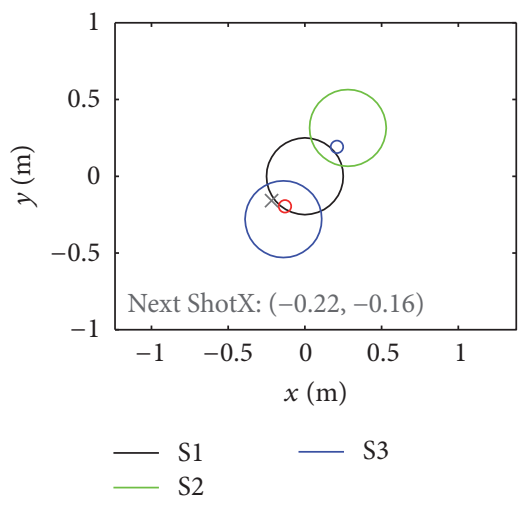

(g)

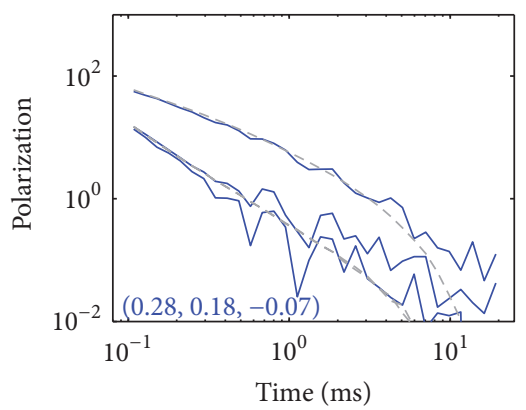

(b)

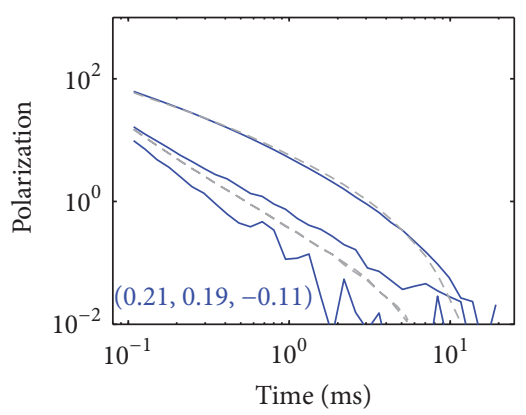

(e)

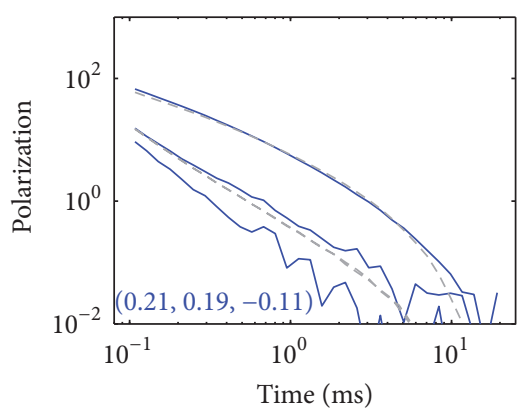

(h)

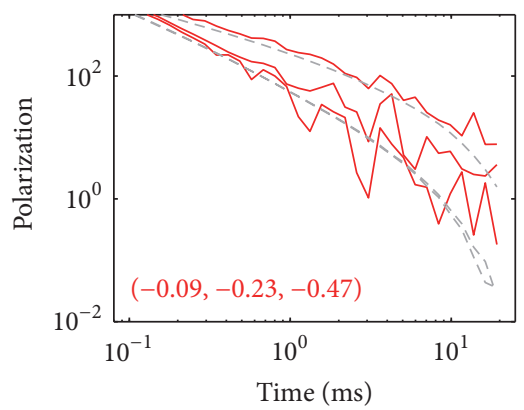

(c)

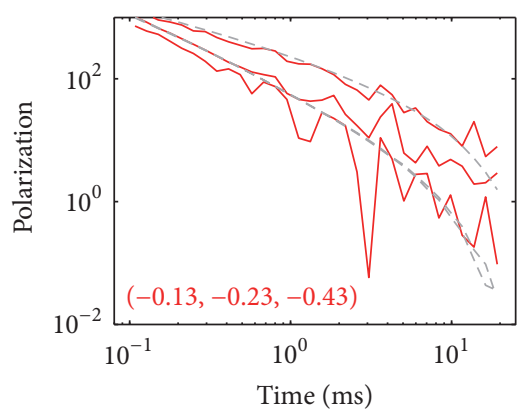

(f)

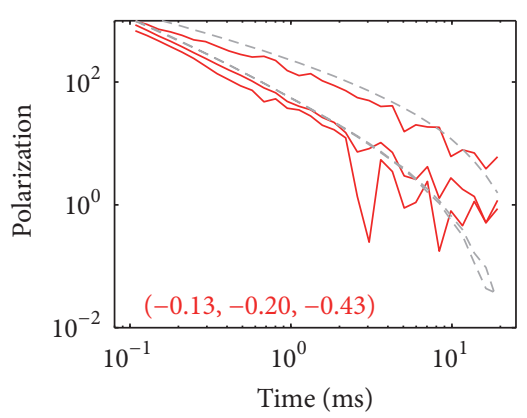

(i)

FIGURE 8: SED experiment with the MPV, two-object example. Initial sounding (S1): (a) the sensor footprint (black circle) and inverted object locations (small circles); (b)-(c) recovered polarizabilities in blue and red and the reference ones in gray dashed. Soundings S1 + S2: (d) the sensor footprint and inverted object locations; (e)-(f) recovered polarizabilities. Soundings S1 + S2 + S3: (g) the sensor footprint and inverted object locations; (h)-(i) recovered polarizabilities.

of 2 , although the SED suggested the third sounding at $(0.26$, $0.35) \mathrm{m}$, a position that would cover the small ISO.

\section{Conclusions}

In this work, we have investigated the application of the experiment design approach to guiding a sensor placement that aims to achieve optimal measurements in practice. The experimental design theory includes the two sets of parameters of interest: the model parameters (locations of targets and their polarizabilities) and design parameter (sensing location). Since determining the optimal design parameter depends on the model parameters that are unknown before an experiment, we adopted the sequential experimental design (SED) technique. By the linearized model covariance matrix that quantifies model uncertainty, an information gain function (a variant of the $D$-criterion) can be defined as a measure of model uncertainty reduction between previous and future surveys. The SED approach recursively solves the target parameters by minimizing the data misfit and forecast the next desired sensor location by maximizing the information gain function. The sequential experimental 


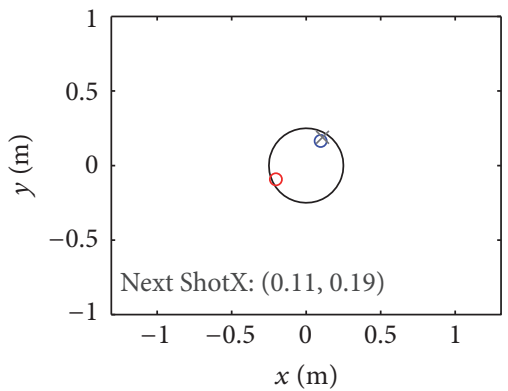

- S1

(a)

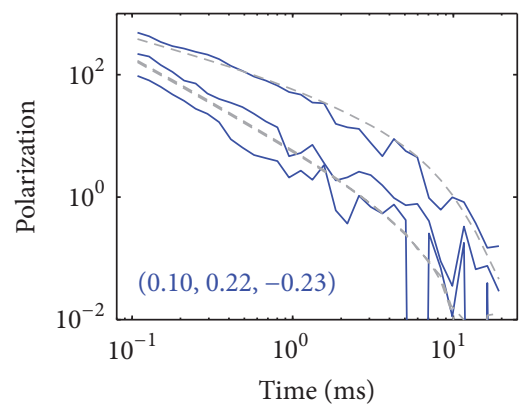

(d)

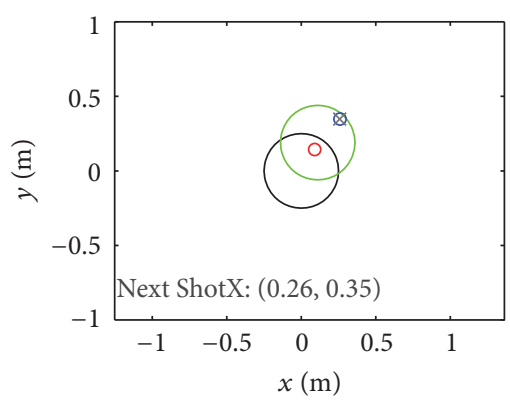

$-\mathrm{S} 1$

$-\mathrm{S} 2$

(g)

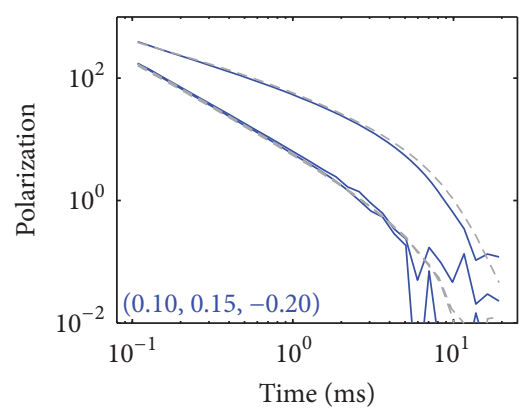

(j)

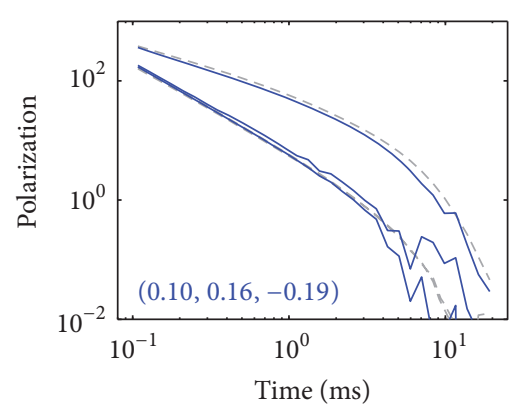

(b)

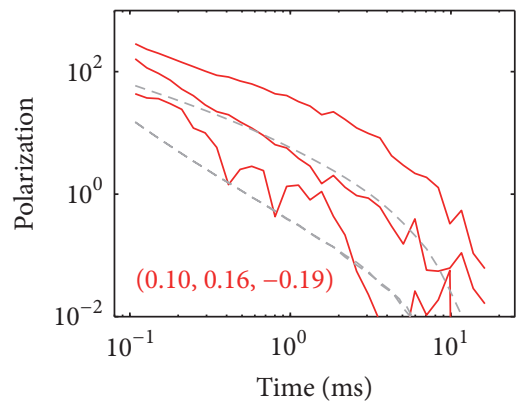

(e)

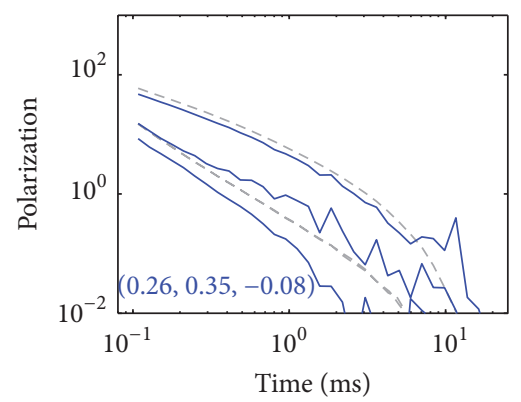

(h)

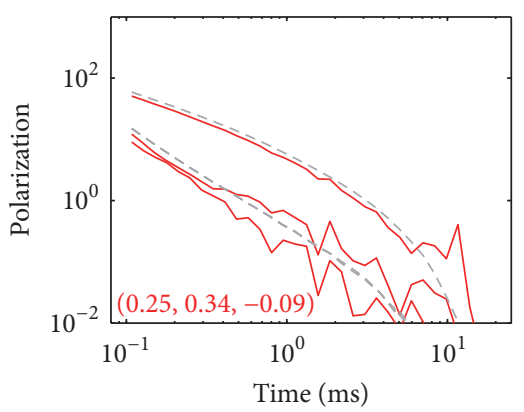

(k)

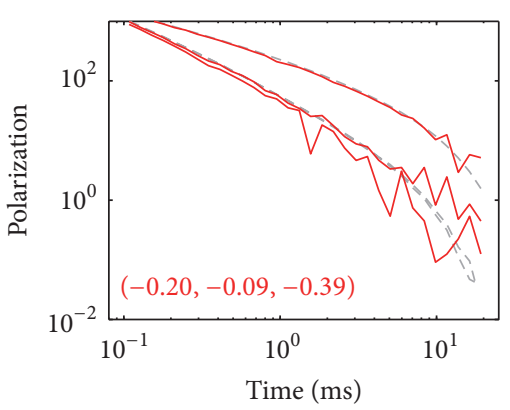

(c)

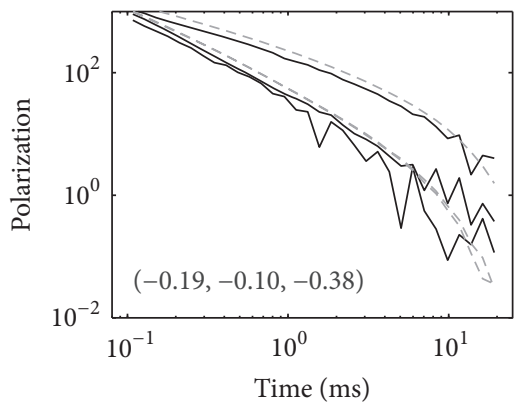

(f)

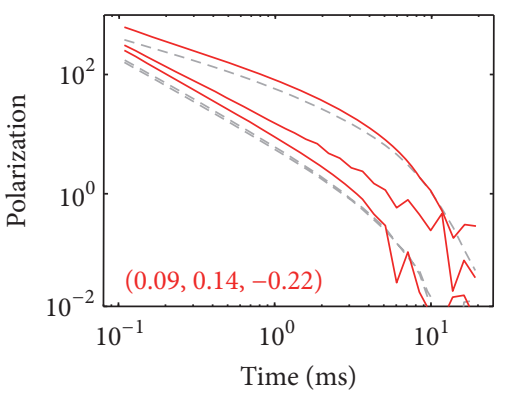

(i)

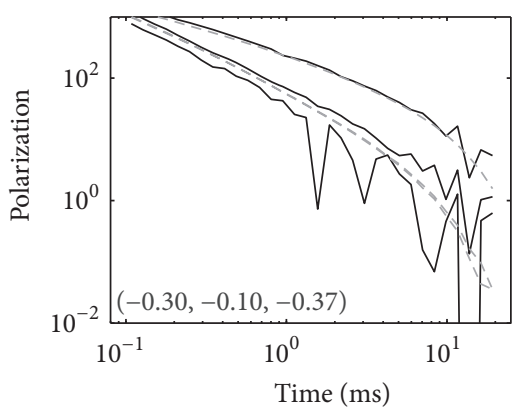

(1)

FIGURE 9: SED experiment with the MPV, three-object example. Initial sounding (S1): (a) the sensor footprint (black circle) and inverted two-object locations (small circles); (b)-(c) recovered polarizabilities with a two-object model; (d)-(f) recovered polarizabilities with a threeobject model. Soundings S1 + S2: (g) the sensor footprint and inverted two-object locations; (h)-(i) recovered polarizabilities with a two-object model; (j)-(l) recovered polarizabilities with a three-object model. Recovered polarizabilities are in blue, red, and black and the reference ones in gray dashed. 
design algorithm is presented in a generic form which allows to incorporate available prior information about model and data into the process through the covariance matrices. In this preliminary study, we consider the simple case assuming $C_{0}^{-1}=0$ and a non-uniform diagonal data covariance matrix.

The synthetic and real tests show that the SED can be a promising tool that can help guide a sensor placement. We further studied the impact of the unknown number of targets on the SED performance. In the TEMTADS2x 2 experiments with a single near sensor-edge object, we observed that the SED is able to suggest a new sounding location that covers the true anomaly under an overestimated 2-object model. After adding one-step guided sensing, the polarizabilities of the actual anomaly were much better recovered than those with the initial sounding. Meanwhile the polarizabilities of the false anomaly become small, remain noisy, and are negligible. However for the case of two objects that are close to sensor edges, an underestimated single-object model could mislead the SED to focus on only one of the targets. As a result, the addition of soundings through the SED brought no improvement to polarizability recovery. In the real MPV experiment with a 3-object case, assuming only two objects for the initial inversion worked for the SED to predict an informative sensing location. The reason was that both the initial sounding and the SED-suggested sounding mainly sensed two objects while the third object remained fairly far from the field view for each survey location. However, inverting the two sets of data simultaneously required a threeobject model to account for the signal contribution of each of the three buried items. As a general rule, an appropriate overrepresentation model in the SED appears to be workable to deal with an unknown number of objects.

In the future, it could be interesting to investigate a noise statistical model that properly specifies correlated noise in EMI measurements. Then for measurement errors known to be correlated, a nondiagonal data covariance matrix can be used to take these correlations into account in the optimal experimental design for the sensor placement problem.

\section{Competing Interests}

The authors declare that there is no conflict of interests regarding the publication of this paper.

\section{Acknowledgments}

The authors are grateful to Drs. Nevin Amin and Sebastian Heidenreich for their efforts to coordinate the review process. This work was supported by a SERDP grant MR-2318.

\section{References}

[1] World Health Organization, Guidance for Surveillance of Injuries Due to Landmines and Unexploded Ordnance, World Health Organization, Geneva, Switzerland, 2000.

[2] B. Delaney and D. Etter, Report of the Defense Science Board Task Force on Unexploded Ordnance, Office of the Under Secretary of Defense For Acquisition, Technology, and Logistics, Washington, DC, USA, 2003.
[3] A. Andrews and H. Nelson, "Implementing advanced classification on munitions response sites: a guide to informed decision making for project managers, regulators, and contractors," ESTCP, 2011.

[4] J. D. Jackson, Classical Electrodynamics, Wiley, New York, NY, USA, 2nd edition, 1975.

[5] F. S. Grant and G. F. West, Interpretation Theory in Applied Geophysics, section 6.5, McGraw-Hill, 1965.

[6] L. R. Pasion and D. W. Oldenburg, "A discrimination algorithm for UXO using time domain electromagnetics," Journal of Environmental and Engineering Geophysics, vol. 6, no. 2, pp. 91102, 2001.

[7] J. T. Smith and H. F. Morrison, "Estimating equivalent dipole polarizabilities for the inductive response of isolated conductive bodies," IEEE Transactions on Geoscience and Remote Sensing, vol. 42, no. 6, pp. 1208-1214, 2004.

[8] L.-P. Song, D. W. Oldenburg, L. R. Pasion, and S. D. Billings, "Transient electromagnetic inversion for multiple targets," in Proceedings of the Detection and Sensing of Mines, Explosive Objects, and Obscured Targets XIV, vol. 7303, Orlando, Fla, USA, April 2009.

[9] T. M. Grzegorczyk, B. E. Barrowes, F. Shubitidze, J. P. Fernández, and K. O'Neill, "Simultaneous identification of multiple unexploded ordnance using electromagnetic induction sensors," IEEE Transactions on Geoscience and Remote Sensing, vol. 49, no. 7, pp. 2507-2517, 2011.

[10] L.-P. Song, L. R. Pasion, S. D. Billings, and D. W. Oldenburg, "Nonlinear inversion for multiple objects in transient electromagnetic induction sensing of unexploded ordnance: technique and applications," IEEE Transactions on Geoscience and Remote Sensing, vol. 49, no. 10, pp. 4007-4020, 2011.

[11] V. V. Fedorov, Theory of Optimal Experiments, Academic Press, New York, NY, USA, 1972.

[12] A. C. Atkinson, A. N. Doney, and R. Tobias, Optimum Experimental Designs, with SAS, Oxford Science Publications, Oxford, UK, 2007.

[13] D. J. C. MacKay, "Information-based objective functions for active data selection," Neural Computation, vol. 4, no. 4, pp. 590604, 1992.

[14] X. Liao and L. Carin, "Application of the theory of optimal experiments to adaptive electromagnetic-induction sensing of buried targets," IEEE Transactions on Pattern Analysis and Machine Intelligence, vol. 26, no. 8, pp. 961-972, 2004.

[15] Y. Das, J. E. McFee, J. Toews, and G. C. Stuart, "Analysis of an electromagnetic induction detector for real-time location of buried objects," IEEE Transactions on Geoscience and Remote Sensing, vol. 28, no. 3, pp. 278-288, 1990.

[16] T. H. Bell, B. J. Barrow, and J. T. Miller, "Subsurface discrimination using electromagnetic induction sensors," IEEE Transactions on Geoscience and Remote Sensing, vol. 39, no. 6, pp. 1286-1293, 2001.

[17] A. Tarantola, Inverse Problem Theory and Methods for Model Parameter Estimation, Society for Industrial and Applied Mathematics (SIAM), Philadelphia, Pa, USA, 2005.

[18] C. D. Meyer, Matrix Analysis and Applied Linear Algebra, SIAM, 2000.

[19] L. R. Pasion, Inversion of time domain electromagnetic data for the detection of unexploded ordnance [Ph.D. dissertation], The University of British Columbia, Vancouver, Canada, 2007.

[20] J. J. Moré, “The Levenberg-Marquardt algorithm: implementation and theory," in Numerical Analysis, G. A. Watson, Ed., 
vol. 630 of Lecture Notes in Mathematics, pp. 105-116, Springer, Berlin, Germany, 1978.

[21] T. F. Coleman and Y. Li, "An interior trust region approach for nonlinear minimization subject to bounds," SIAM Journal on Optimization, vol. 6, no. 2, pp. 418-445, 1996.

[22] Optimization Toolbox Users Guide, The Math Works Inc., Natick, Mass, USA, 2013.

[23] ESTCP MR-201165 Demonstration Data Report Former Camp Beale TEMTADS MP 2x2 Cart Survey, 2011.

[24] J. P. Fernandez, B. E. Barrowes, T. M. Grzegorczyk, N. Lhomme, K. O’Neill, and F. Shubitidze, "A man-portable vector sensor for identification of unexploded ordnance," IEEE Sensors Journal, vol. 11, no. 10, pp. 2542-2555, 2011.

[25] K. A. Kingdon, N. Lhomme, and D. C. George, "A man portable EMI system for detection and classification of unexploded ordnance in challenging environments," FastTimes, vol. 19, no. 3, pp. 31-41, 2014. 


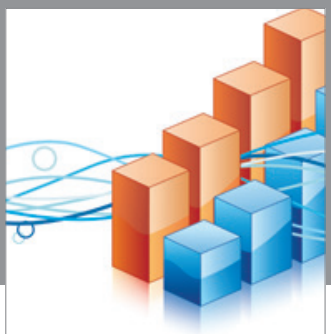

Advances in

Operations Research

vatem alat4

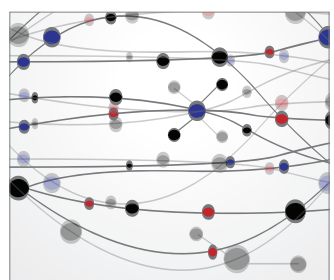

\section{The Scientific} World Journal
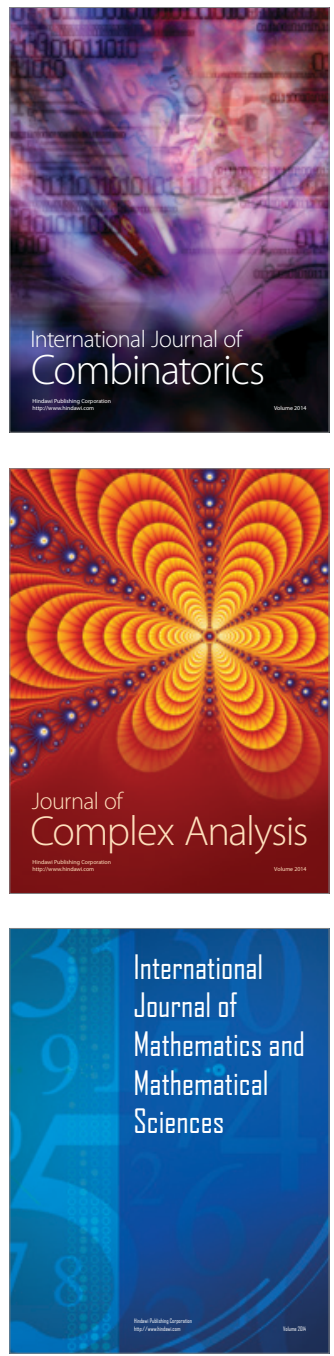
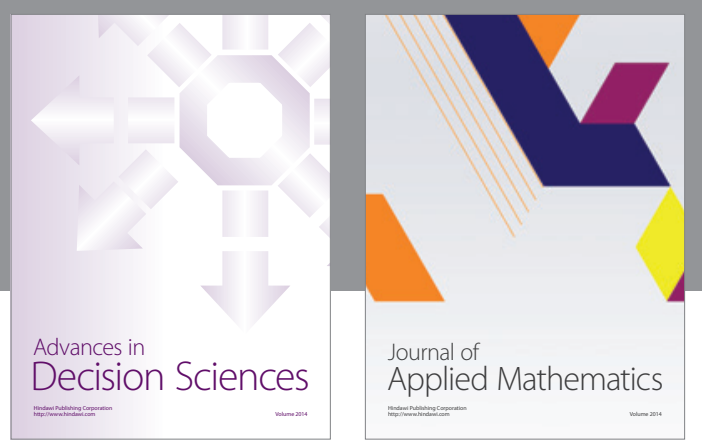

Algebra

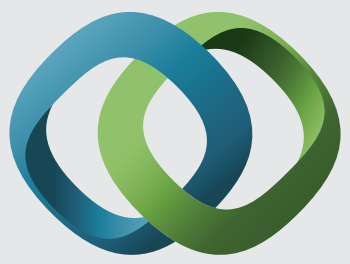

\section{Hindawi}

Submit your manuscripts at

http://www.hindawi.com
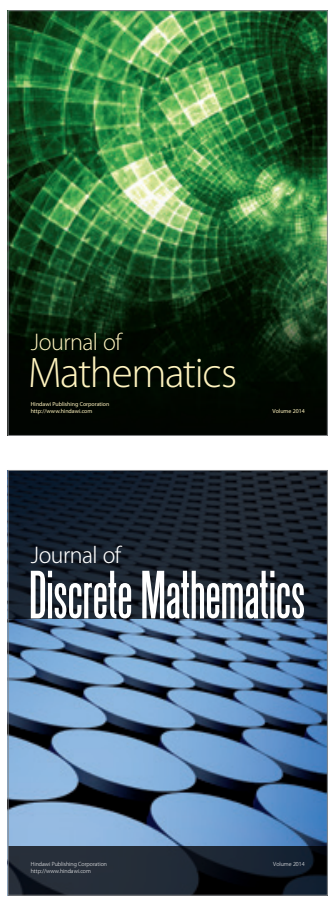

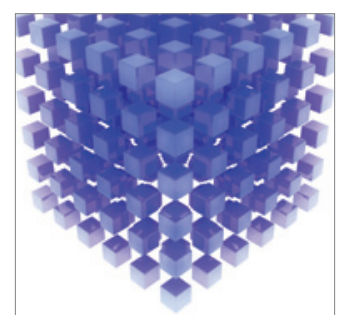

Mathematical Problems in Engineering
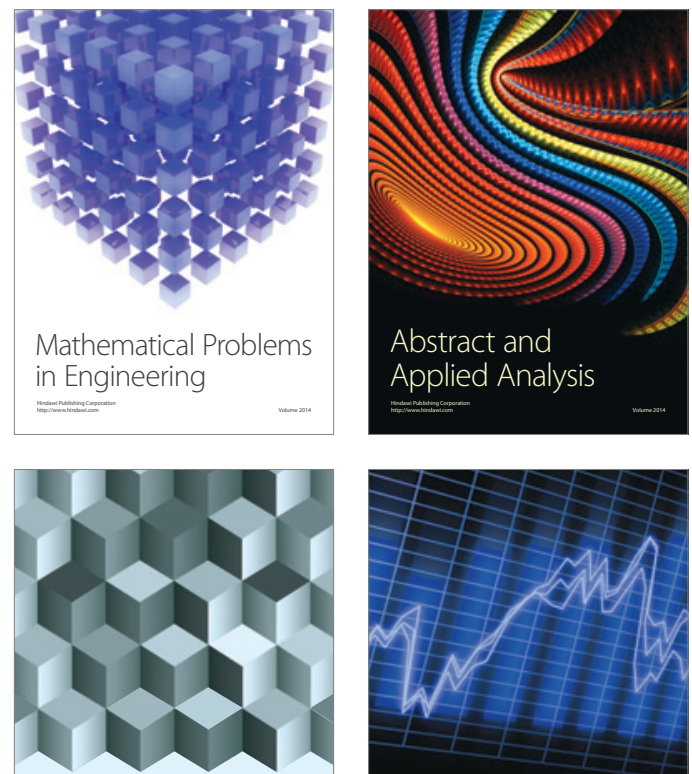

Journal of

Function Spaces

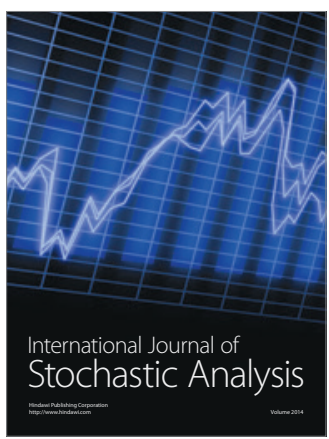

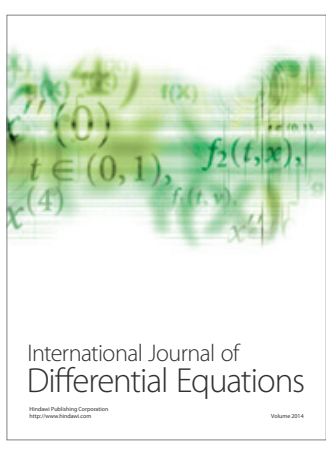
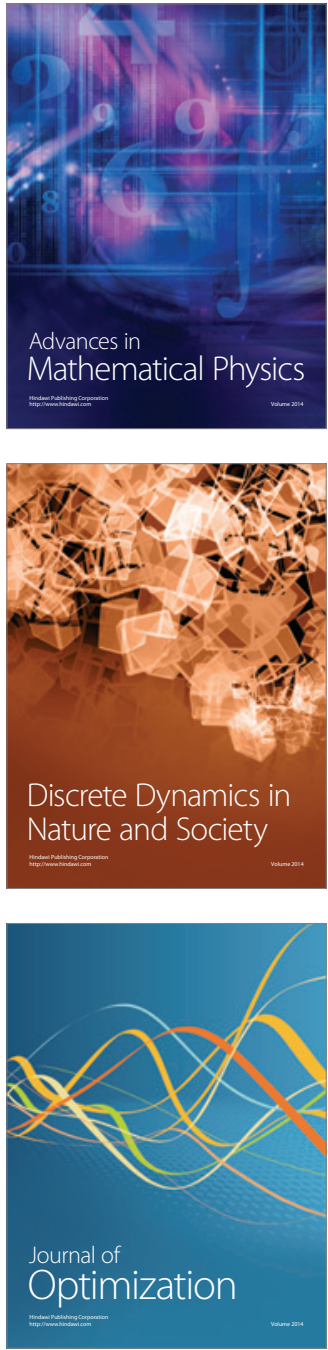